\title{
Transient analysis including the low- and the medium-frequency ranges of engineering structures
}

\author{
Mathilde Chevreuil *, Pierre Ladevèze, Philippe Rouch \\ LMT Cachan (ENS Cachan, CNRS, Paris 6 University), 61 avenue du Président Wilson, 94235 Cachan Cedex, France
}

\begin{abstract}
This paper deals with a computational method for transient dynamic analysis which enables one to cover both the low- and mediumfrequency ranges. This is a frequency approach in which the low-frequency part is obtained through a classical technique while the medium-frequency part is handled through the Variational Theory of Complex Rays (VTCR) initially introduced for vibrations. The aim of this paper is to show the capabilities of the method for transient analyses of complex engineering structures submitted to impact loadings.
\end{abstract}

Keywords: Transient dynamics; Frequency domain; Medium frequencies; Computation; VTCR; Multiscale

\section{Introduction}

The design of industrial structures requires engineers to know their dynamic behavior. The response, especially during the transient stage, cannot be completely described using current tools based on finite element techniques and explicit numerical schemes; indeed, the medium-frequency range is often ignored unless the calculation is carried out with a very refined spatial mesh and, consequently, a refined time discretization $[1,2]$. This would mean a prohibitive computation time. But taking the medium-frequency content into account can prove necessary since, although the displacements are small over this frequency range, the velocity and therefore the kinetic energy can be significant. Transient dynamic analysis in this frequency range for complex engineering structures presents an important challenge. An answer is given by the new computational strategy introduced in [3] and developed here. The problem being solved in the frequency domain, one needs to solve a forced vibration problem over a frequency range which includes the low- and medium-frequency

\footnotetext{
${ }^{*}$ Corresponding author. Tel.: +331474028 75/41; fax: +331474022 40.

E-mail address: mathilde.chevreuil@Imt.ens-cachan.fr (M. Chevreuil).
}

ranges. The low-frequency range is solved as usual while the medium-frequency range is handled using the Variational Theory of Complex Rays (VTCR). The final solution in the space-time domain is given using the inverse Fourier transform.

The main problem resides in the resolution of the forced vibration problem over a wide-frequency range. The lowfrequency range no longer poses any major difficulties, at least regarding modeling and calculation, even for complex structures. As for high frequencies, computational tools quite different from those used for low frequencies are available, in particular the SEA method in which the spatial aspects disappear almost entirely [4].

By contrast, the modeling and calculation of mediumfrequency vibrations, on which this paper focuses, continue to raise some problems. The difficulty lies in the fact that the wavelength of the phenomenon being studied is very small compared to the characteristic dimension of the structure. Consequently, if one were to extend the low-frequency methods disregarding the serious numerical difficulties which would occur, the corresponding finite element calculation would still require an unreasonable number of degrees of freedom. This situation would be made even worse by the pollution error due to the extended range of calculated frequencies which would affect the accuracy of 
the finite element solution [5,6]. Different remedies for that problem have been tried, such as enhanced finite elements [7-9], specific reduced bases [10-12] or a combination of a wave-based method with a Trefftz approach [13], but most of these techniques require very fine meshes. Difficulties are also experienced when one attempts, as in [14-17], to extend the SEA method (which is appropriate for high frequencies) because most of these methods require additional information (e.g. coupling loss factors) and specific geometries.

The alternative approach we use here, called the "Variational Theory of Complex Rays", was introduced in [18] for the calculation of medium-frequency vibrations. This approach, whose main limitation is that the structure must lend itself to partitioning into homogeneous substructures, is defined as follows.

The first characteristic of this approach is the use of a new variational formulation of the problem being considered (i.e. forced vibrations at a given frequency) which enables one to use a priori independent approximations within each substructure. In other words, the transmission conditions on the displacements as well as the stresses at the interfaces between substructures do not need to be verified a priori, but are built in the variational formulation.

The second characteristic of the VTCR is the introduction within each substructure of two-scale approximations with a strong mechanical meaning: the solution is assumed to be well-described as the superposition of an infinite number of local vibration modes. These basic modes (which can be interior modes, boundary modes or corner modes) verify the law of dynamics. All wave directions are taken into account and the unknowns are discretized amplitudes with relatively large wavelengths.

Thus, the proposed approach to the calculation of the transient dynamic response consists in dividing the frequency range being studied into two parts: the low-frequency range, over which the frequency response function is obtained with a standard finite element technique, and the medium-frequency range, in which the suitable method to calculate the frequency response function is the VTCR [18]. This paper recalls only the basic aspects of the Variational Theory of Complex Rays.

The proposed approach, which is an extension of the VTCR (initially introduced for medium-frequency forced vibration problems) to transient dynamics, has been applied on beams in $[3,19]$ and shows the importance of the medium frequencies. The aim of this paper is to develop this method to complex engineering structures as assemblies of plates, shells and beams submitted to an impact. To study such structures, a first difficulty is to be able to compute at low cost with the VTCR over a rather large frequency range; what has been proposed in [20] is applied here. Another important point concerns the calculation of the time response through the inverse Fourier transform which could be quite costly with the usual direct technique. Here, we propose an enhanced technique where the time response is described as the superposition of two components that stem respectively from the low- and the mid-frequency contributions. Each component is determined separately. The time response related to the low-frequency part does not involve any difficulty; it is done using the classical technique. For the time response of the medium-frequency part, we have developed a technique taking advantage of the quick fading-out of medium-frequency vibrations in scattering media. That reduces strongly the computational cost.

The main part of the paper deals with an application of the method to a rather complex structure and then to show the capability of the method to carry out computations on complex engineering structures (assemblies of plates, shells and beams, etc.) submitted to impact loads.It also displays the importance of the medium-frequency part transient analyses.

\section{The dynamic reference problem}

Let us consider, under the assumptions of small perturbations, the dynamic equilibrium of a structure defined in the space domain $\Omega$, and let $\partial \Omega$ be the boundary of $\Omega$. At each time $t$ of the interval $[0, T]$ being studied, this structure is subjected to the following actions:

- a displacement field $\underline{U}_{d}$ on a portion $\partial_{1} \Omega$ of boundary $\partial \Omega$,

- a force density $\underline{F}_{d}$ on the portion $\partial_{2} \Omega$ of $\partial \Omega$ which is the complementary part of $\partial_{1} \Omega$,

- a force density $f_{d}$ on the whole domain $\Omega$.

For every $\underline{M}$ belonging to $\Omega$, the displacements are subjected to initial conditions at $t=0$ :

$\underline{U}_{\mid t=0}=\underline{U}_{0}$

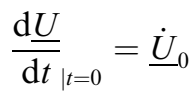

where $\underline{U}_{0}$ and $\underline{\dot{U}}_{0}$ are given.

Let us also define for the structure $\Omega$ the constitutive relation:

$\boldsymbol{\sigma}=\mathbf{K} \boldsymbol{\varepsilon}(\underline{U})+\eta \mathbf{K} \dot{\boldsymbol{\varepsilon}}(\underline{U})$

where $\mathbf{K}$ is the Hooke's operator. In the present work, damping is introduced classically in terms of the frequency with $\eta>0$. More complex constitutive relations could also be taken into account.

The reference frame is assumed to be Galilean. The reference problem of the evolution of the structure during $[0, T]$ can be formulated as follows: Find $\underline{U}(\underline{M}, t) \in \mathscr{U}^{[0, T]}$ and $\boldsymbol{\sigma}(\underline{M}, t) \in \mathscr{S}^{[0, T]}$, with $\underline{M} \in \Omega$ and $t \in[0, T]$, which verify:

- the compatibility equations and the initial conditions:

$$
\begin{aligned}
& \underline{U}_{\partial_{1} \Omega}=\underline{U}_{d} \\
& \underline{U}_{\mid t=0}=\underline{U}_{0}, \quad \frac{\mathrm{d} \underline{U}}{\mathrm{~d} t}{ }_{\mid t=0}=\underline{\dot{U}}_{0}
\end{aligned}
$$


- the dynamic equilibrium equation:

$$
\begin{aligned}
\forall t & \in] 0, T\left[, \quad \forall \underline{\dot{U}}^{*} \in \mathscr{U}_{0}, \quad \int_{\Omega} \rho \frac{\mathrm{d}^{2} \underline{U}}{\mathrm{~d} t^{2}} \cdot \underline{\dot{U}}^{*} \mathrm{~d} \Omega\right. \\
& =-\int_{\Omega} \operatorname{Tr}\left[\boldsymbol{\sigma} \boldsymbol{\varepsilon}\left(\dot{U}^{*}\right)\right] \mathrm{d} \Omega+\int_{\Omega} \underline{f}_{d} \cdot \underline{\dot{U}}^{*} \mathrm{~d} \Omega+\int_{\partial_{2} \Omega} \underline{F}_{d} \cdot \dot{U}^{*} \mathrm{~d} S
\end{aligned}
$$

- the constitutive relation:

$$
\boldsymbol{\sigma}=\mathbf{K} \boldsymbol{\varepsilon}(\underline{U})+\eta \mathbf{K} \dot{\boldsymbol{\varepsilon}}(\underline{U})
$$

$\mathscr{U}_{0}$ is the subspace of $\mathscr{U}^{[0, T]}$ associated with a zero value of $\underline{U}_{d}$ on boundary $\partial_{1} \Omega$.

\section{Frequency analysis of the reference problem}

\subsection{Frequency formulation of the problem}

Let us rewrite the transient dynamic problem as a global variational problem over the frequency-space domain. Thus, the Fourier transform is applied to all time-dependent quantities, yielding frequency-dependent functions:

$\hat{f}(\omega)=\int_{-\infty}^{+\infty} f(t) \mathrm{e}^{-\mathrm{i} \omega t} \mathrm{~d} t$

Thus, the reference problem can be reformulated as follows: Find $(\underline{\hat{U}}(\underline{M}, \omega), \hat{\boldsymbol{\sigma}}(\underline{M}, \omega))$, with $\underline{M} \in \Omega$ and $\omega \in \mathbb{R}$, which verify:

- the compatibility equations:

$$
\begin{aligned}
& \underline{\hat{U}}_{\partial_{1} \Omega}=\underline{\hat{U}}_{d} \\
& \underline{\hat{U}}=\mathrm{i} \omega \underline{\hat{U}}
\end{aligned}
$$

- the dynamic equilibrium equation:

$$
\begin{aligned}
\forall \omega \in & \mathbb{R}, \quad \forall \underline{\hat{U}}^{*} \in \mathscr{U}_{0}, \quad \int_{\Omega}-\rho \omega^{2} \underline{\hat{U}} \cdot \underline{\hat{U}}^{*} \mathrm{~d} \Omega \\
= & -\int_{\Omega} \operatorname{Tr}\left[\hat{\boldsymbol{\sigma}} \varepsilon\left(\hat{U}^{*}\right)\right] \mathrm{d} \Omega+\int_{\Omega} \underline{\hat{f_{d}}} \cdot \underline{\hat{U}}^{*} \mathrm{~d} \Omega \\
& +\int_{\hat{\mathrm{O}}_{2} \Omega} \underline{\hat{F}}_{d} \cdot \underline{\hat{U}}^{*} \mathrm{~d} S
\end{aligned}
$$

- the constitutive relation:

$$
\hat{\boldsymbol{\sigma}}=(1+\mathrm{i} \eta) \mathbf{K} \boldsymbol{\varepsilon}(\underline{\hat{U}})
$$

Putting the accent on the displacement, the reference problem can be rewritten as: Find $\underline{\hat{U}}(\underline{M}, \omega)$, with $\underline{M} \in \Omega$ and $\omega \in \mathbb{R}$, such that:

$$
\begin{aligned}
& \hat{U}_{\partial_{1} \Omega}=\hat{U}_{d} \\
& \forall \omega \in \mathbb{R}, \quad \forall \underline{\hat{U}}^{*} \in \mathscr{U}_{0} \\
& \quad \int_{\Omega}\left\{-\rho \omega^{2} \underline{\hat{U}} \cdot \underline{\hat{U}}^{*}+(1+\mathrm{i} \eta) \operatorname{Tr}\left[\mathbf{K} \boldsymbol{\varepsilon}(\underline{\hat{U}}) \boldsymbol{\varepsilon}\left(\underline{\hat{U}}^{*}\right)\right]\right\} \mathrm{d} \Omega \\
& \quad=\int_{\Omega} \underline{\hat{f}_{d}} \cdot \underline{\hat{U}}^{*} \mathrm{~d} \Omega+\int_{\partial_{2} \Omega} \underline{\hat{F}}_{d} \cdot \underline{\hat{U}}^{*} \mathrm{~d} S
\end{aligned}
$$

For a given $\omega$, the previous problem is a forced vibration problem whose solution can easily be shown to be unique. Using the frequency approach, one needs to solve the forced vibration problem over a wide-frequency range $\left[0, \omega_{c}^{\prime}\right]$ which contains the low- and medium-frequency ranges; finally, one must calculate the frequency response function $\underline{\hat{h}}(\omega)$ of the system for $\omega \in\left[0, \omega_{c}^{\prime}\right]$.

\subsection{Principle of the new computational approach}

The present approach considers a partition of the frequency range $\left[0, \omega_{c}^{\prime}\right]$ being studied into two parts:

- a low-frequency part $\left[0, \omega_{c}\right]$,

- a medium-frequency part $\left[\omega_{c}, \omega_{c}^{\prime}\right]$.

Outside of this frequency range, the kinetic and strain energies in the structure are assumed to be negligible as it will be shown in Section 5.3.2.

For the low-frequency range $\left[0, \omega_{c}\right]$, the frequency response function is obtained using a finite element technique: it is advantageous to use a reduced basis constructed from the first vibration modes and completed with the static modes. With this very standard approach (see e.g. $[1,21])$, the displacement can be written as

$\underline{\hat{U}}(\underline{M}, \omega)=\sum_{i=1}^{n} a_{i}(\omega) \underline{\varphi_{i}}(\underline{M})+\sum_{j=1}^{m} b_{j}(\omega) \underline{\varphi}_{j}^{\prime}(\underline{M})$

where $\underline{\varphi}_{i}(\underline{M})$ are the eigenmodes of the structure and $\underline{\varphi}_{j}^{\prime}(\underline{M})$ are the static modes. The basis contains at least all the eigenvectors $\underline{\varphi}_{i}(\underline{M})$ such that $\omega_{i} \leqslant 2: \omega_{c}$. The $\underline{\varphi}_{i}(\underline{M}), i \in$ $\{1,2, \ldots, n\}$ and $\varphi_{j}^{\prime}(\underline{M}), j \in\{1,2, \ldots, m\}$ are constructed in such a way that they are orthogonal with respect to the kinetic energy.

The Variational Theory of Complex Rays (VTCR), which will be presented in Section 4, is a suitable computational method for the medium-frequency range $\left[\omega_{c}, \omega_{c}^{\prime}\right]$.

For $\omega^{-} \leqslant 0$, the frequency response is the conjugate of the response with respect to the excitation's frequency $\omega^{+}=-\omega^{-}$.

\subsection{Back to the time response}

After the frequency response functions of the points of interest in the structure have been calculated over $\left[0, \omega_{c}^{\prime}\right]$, the time response is restored using the inverse Fourier transform:

$f(t)_{t \geqslant 0}=\frac{1}{2 \pi} \int_{-\infty}^{+\infty} \hat{f}(\omega) \mathrm{e}^{\mathrm{i} \omega t} \mathrm{~d} \omega$

\section{Outline of the VTCR for the resolution of the forced vibration problem for the medium-frequency part}

Only the basic aspects are given here. More details can be found in [22-24]. 


\subsection{The reference problem}

In order to simplify the presentation, we present the problem for an assembly of two substructures, but this formulation can be easily generalized to an assembly of $n$ substructures. Given two substructures $S$ and $S^{\prime}$, let $\partial S$ and $\partial S^{\prime}$ be the boundaries of $S$ and $S^{\prime}$ respectively. We are studying the harmonic vibration of these two structures at a fixed frequency $\omega$. All quantities can be defined in the complex domain: an amplitude $Q(\underline{M})$ is associated with $Q(\underline{M}) \mathrm{e}^{\mathrm{i} \omega t}$.

The excitations applied to $S$ and shown in Fig. 1 are

- a displacement field $\underline{U}_{d}$ on a portion $\partial_{U} S$ of the boundary $\partial S$,

- a force density $\underline{F}_{d}$ on a portion $\partial_{F} S$ of $\partial S$,

- a force density $f_{d}$ on the whole domain $S$,

where $\underline{U}_{d}, \underline{F}_{d}, f_{d}$ are the amplitudes of the quantities defined in the complex domain.

Similar quantities are defined for $S^{\prime}$.

Let us define for structure $S$ the displacement-stress pair $s=(\underline{U}, \boldsymbol{\sigma})$ and the corresponding space $\mathscr{S}_{a d}$ such that:

$\begin{array}{ll}\underline{U} \in \mathbf{U} & \text { (finite-energy displacement set }\left[H^{1}(S)\right]^{3} \text { ) } \\ \boldsymbol{\sigma} \in \mathbf{S} & \left.\text { (finite-energy stress set }\left[L^{2}(S)\right]^{3}\right) \\ \underline{\gamma}=\underline{\operatorname{div} \boldsymbol{\sigma}+\underline{f}_{d}} & \text { on } S \\ \boldsymbol{\sigma}=(1+\mathrm{i} \eta) \mathbf{K} \boldsymbol{\varepsilon}(\underline{U}) & \text { on } S \\ \underline{\gamma}=-\omega^{2} \rho \underline{U} & \text { on } S\end{array}$

where $\mathbf{K}$ is the Hooke's operator, $\rho$ the mass density, $\eta$ the damping coefficient (which can depend on the frequency) and $\chi$ is the acceleration. The subspace of $\mathscr{S}_{a d}$ associated with a zero value of $f_{d}$ is denoted $\mathscr{S}_{a d, 0}$. Similarly, we introduce spaces $\mathscr{S}_{a d}^{\prime}$ and $\mathscr{S}_{a d, 0}^{\prime}$.

The reference problem can be formulated as follows: Find $(\underline{U}(\underline{M}), \sigma(\underline{M}), \underline{M} \in S)$ and $\left(\underline{U^{\prime}}(\underline{M}), \sigma^{\prime}(\underline{M}), \underline{M} \in S^{\prime}\right)$ such that

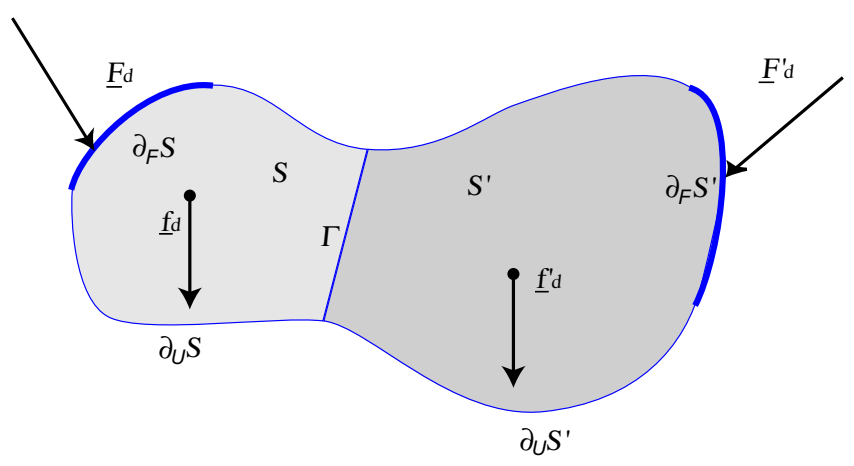

Fig. 1. The reference problem.

$$
\begin{aligned}
& s=(\underline{U}, \boldsymbol{\sigma}) \in \mathscr{S}_{a d}, \quad s^{\prime}=\left(\underline{U}^{\prime}, \boldsymbol{\sigma}^{\prime}\right) \in \mathscr{S}_{a d}^{\prime} \\
& \underline{U}=\underline{U}_{d} \quad \text { on } \partial_{U} S, \quad \underline{U}^{\prime}=\underline{U}_{d}^{\prime} \quad \text { on } \partial_{U} S^{\prime} \\
& \underline{\boldsymbol{\sigma}}=\underline{F}_{d} \quad \text { on } \partial_{F} S, \quad \boldsymbol{\sigma}^{\prime} \underline{n}^{\prime}=\underline{F}_{d}^{\prime} \quad \text { on } \partial_{F} S^{\prime} \\
& \underline{U}=\underline{U}^{\prime} \quad \text { on } \Gamma \\
& \underline{\boldsymbol{\sigma}}+\underline{\boldsymbol{\sigma}}^{\prime} \underline{\prime}^{\prime}=\underline{0} \quad \text { on } \Gamma
\end{aligned}
$$

\subsection{The variational formulation associated with the VTCR}

The VTCR is a global formulation of the boundary conditions (14) in terms of both displacements and forces. All boundary conditions on the edges of the substructures can be taken into account thanks to the following variational formulation. It is based on a priori independent approximations within the substructures: Find $s=(\underline{U}, \boldsymbol{\sigma}) \in \mathscr{S}_{a d}$ and $s^{\prime}=\left(\underline{U}^{\prime}, \boldsymbol{\sigma}^{\prime}\right) \in \mathscr{S}_{a d}^{\prime}$ such that

$$
\begin{aligned}
\operatorname{Re} & \left\{-\mathrm{i} \omega\left[\int_{\partial_{U} S} \delta \boldsymbol{\sigma} \underline{n}\left(\underline{U}^{*}-\underline{U}_{d}^{*}\right) \mathrm{d} l+\int_{\partial_{F} S}\left(\boldsymbol{\sigma} \underline{n}-\underline{F}_{d}\right) \delta \underline{U}^{*} \mathrm{~d} l\right.\right. \\
& +\int_{\partial_{U} S^{\prime}} \delta \boldsymbol{\sigma}^{\prime} \underline{n}^{\prime}\left(\underline{U}^{*}-\underline{U}_{d}^{\prime *}\right) \mathrm{d} l+\int_{\partial_{F} S^{\prime}}\left(\boldsymbol{\sigma}^{\prime} \underline{n}^{\prime}-\underline{F}_{d}^{\prime}\right) \delta \underline{U}^{\prime *} \mathrm{~d} l \\
& \left.\left.+\frac{1}{2} \int_{\Gamma}\left(\delta \boldsymbol{\sigma} \underline{n}-\delta \boldsymbol{\sigma}^{\prime} \underline{n}^{\prime}\right)\left(\underline{U}^{*}-\underline{U}^{*}\right)+\left(\boldsymbol{\sigma} \underline{n}-\boldsymbol{\sigma}^{\prime} \underline{n}^{\prime}\right)\left(\delta \underline{U}^{*}-\delta \underline{U}^{\prime *}\right) \mathrm{d} l\right]\right\} \\
& =0 \quad \forall(\delta \underline{U}, \delta \boldsymbol{\sigma}) \in \mathscr{S}_{a d, 0} \forall\left(\delta \underline{U}^{\prime}, \delta \boldsymbol{\sigma}^{\prime}\right) \in \mathscr{Y}_{a d, 0}^{\prime}
\end{aligned}
$$

where $\operatorname{Re}[A]$ designates the real part of a quantity $A$ and $A^{*}$ the conjugate of $A$. It is easy to prove that the variational form is equivalent to the reference problem, provided that:

- the reference problem has a solution,

- the Hooke's operator $\mathbf{K}$ is positive definite,

- the damping coefficients are such that $\eta, \eta^{\prime}>0$.

\subsection{Approximate formulations}

\subsubsection{Principle}

All that is needed in order to derive an approximate formulation from the VTCR is the definition of subspaces $\mathscr{S}_{a d}^{h}$ and $\mathscr{S}_{a d, 0}^{h}\left(\right.$ resp. $\mathscr{S}_{a d}^{\prime h}$ and $\mathscr{S}_{a d, 0}^{\prime h}$ ) from $\mathscr{S}_{a d}$ and $\mathscr{S}_{a d, 0}$ (resp. $\mathscr{S}_{a d}^{\prime}$ and $\mathscr{S}_{a d, 0}^{\prime}$ ) for each substructure. The approximate formulation can be expressed as: Find $s^{h}=\left(\underline{U}^{h}, \boldsymbol{\sigma}^{h}\right) \in \mathscr{S}_{a d}^{h}$ and $s^{\prime h}=\left(\underline{U}^{\prime h}, \sigma^{\prime h}\right) \in \mathscr{S}_{\text {ad }}^{\prime h}$ such that

$$
\begin{aligned}
& \delta\left(E_{D}\left(\underline{U}^{h}\right)+E_{D}^{\prime}\left(\underline{U}^{\prime h}\right)\right)+\left\langle\left[\begin{array}{c}
s^{h} \\
s^{\prime h}
\end{array}\right], \delta\left[\begin{array}{c}
s^{h} \\
s^{\prime h}
\end{array}\right]\right\rangle=\left(L_{D}, \delta\left[\begin{array}{c}
s^{h} \\
s^{\prime h}
\end{array}\right]\right) \\
& \forall \delta s^{h} \in \mathscr{S}_{a d, 0}^{h} \quad \forall \delta s^{\prime h} \in \mathscr{S}_{a d, 0}^{\prime h}
\end{aligned}
$$

where $E_{D}$ is the dissipated power, $L_{D}$ a linear form and $\langle. .$. a bilinear form, defined on the boundary between the substructures, such that $\langle u, v\rangle=-\langle v, u\rangle^{*}$.

The VTCR uses two scales of approximation $\left(U^{h}, \sigma^{h}\right)$, each with a strong mechanical meaning, defined by identifying three zones: the interior zone, the edge zone and the corner zone. For example, in the neighborhood of a point $\underline{X}$ of the interior zone, the solution is assumed to be prop- 
erly described locally as the superposition of an infinite number of local vibration modes which can be written in the following manner:

$$
\begin{aligned}
& \underline{U}^{h}(\underline{X}, \underline{Y}, \underline{P})=\underline{W}^{h}(\underline{X}, \underline{P}) \mathrm{e}^{\mathrm{i} \omega \underline{P} \cdot \underline{Y}} \\
& \sigma^{h}(\underline{X}, \underline{Y}, \underline{P})=C^{h}(\underline{X}, \underline{P}) \mathrm{e}^{\mathrm{i} \omega P \underline{Y} \underline{Y}}
\end{aligned}
$$

where both $\underline{X}$ and $\underline{Y}$ represent the position vector, $\underline{X}$ being associated with slow variations and $\underline{Y}$ with rapid variations. More precisely, the terms related to the position vector $\underline{X}$ vary slowly when $\underline{X}$ moves along the structure, whereas the terms related to the position vector $\underline{Y}$ vary rapidly when $\underline{Y}$ moves along the structure. $\underline{P}$ is a vector characterizing the local vibration mode. In order for these local modes $\left(\underline{U}^{h}, \boldsymbol{\sigma}^{h}\right)$ to be admissible, they must be in $\mathscr{S}_{a d}^{h}$ and verify (13). Thus, we get some properties of $\underline{P}$. These modes verify the interior equations only, without taking the boundary conditions on the edges into account. The cases of ponctual or surface forces are treated in [22].

For instance, let us consider the out-of-plane bending motions of thin, flat, homogeneous and isotropic plates. According to Kirchhoff's thin plate theory, the steady-state displacement $u$ of the plate's mid-surface in the direction perpendicular to the plate is governed by the dynamic equation:

$\frac{E h^{3}}{12\left(1-v^{2}\right)}(1+\mathrm{i} \eta) \Delta \Delta u=\rho h \omega^{2} u$ on $S$

where $\Delta$ is the Laplacian operator, $E$ the Young's modulus, $h$ the plate's thickness, $v$ the Poisson's ratio, $\rho$ the mass density, $\omega$ the frequency, and $\eta$ the damping factor. A complex ray for the interior modes is

$u_{i}^{h}(\underline{X}, \underline{Y}, \underline{P})=w_{i}^{h}(\underline{X}, \underline{P}) \mathrm{e}^{\left(\frac{\eta}{4} \sqrt{\omega} \underline{P} \underline{X}\right)} \mathrm{e}^{\mathrm{i} \sqrt{\omega} \underline{P} \cdot \underline{Y}}$

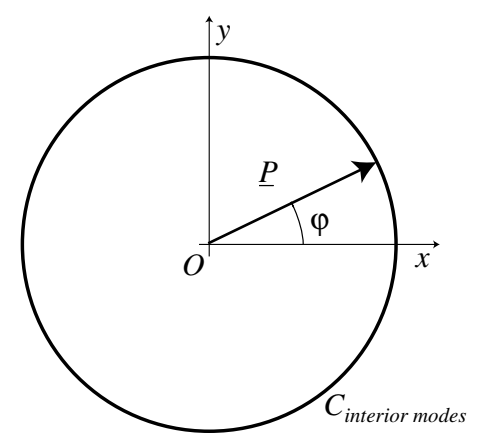

Fig. 2. Admissible $\underline{P}$ for interior modes of an isotropic plate.
This complex ray corresponds to a plane bending wave which propagates through the plate in the $\underline{P}$ direction. This ray is admissible only if

$\frac{E h^{3}}{12\left(1-v^{2}\right)}(1+\mathrm{i} \eta) \Delta_{\underline{X}} \Delta_{\underline{X}} w_{i}^{h}=\rho h \omega^{2} w_{i}^{h} \quad$ on $S$

Therefore, the properties of $\underline{P}$ are

$(\underline{P} \cdot \underline{P})^{2}=r^{4} \quad$ with $r^{4}=\frac{12 \rho\left(1-v^{2}\right)}{E h^{2}}$

Eq. (21) shows that $\underline{P}$ lies on a circle $C$ defined by the material properties (see Fig. 2). All directions of the plate can be taken into account by following this circular path. A similar approach can be used for the edge and corner zones. Examples of such modes are shown in Fig. 3.

\subsubsection{The discretized problem}

The displacement of any point of the substructure is generated by a basis of admissible complex rays. The unknown is the generalized amplitude $w^{h}(\underline{X}, \underline{P})$ of the basis (an $n$ th-order polynomial in $\underline{X}$ and a large-wavelength quantity). This polynomial plays the same role as p-version of the finite element methods and will thereafter be chosen zeroth order [24]. Accounting for all the directions $\varphi$ in $C$ leads to an integral over $C$. For the interior rays, this integral takes the form

$u^{h}(\underline{X}, \underline{Y})=\int_{\varphi \in[0 ; 2 \pi]} w^{h}(\underline{X}, \underline{P}(\varphi)) \mathrm{e}^{\left(\frac{n}{4} \sqrt{\omega} \underline{P}(\varphi) \cdot \underline{X}\right)} \mathrm{e}^{\mathrm{i} \sqrt{\omega} \underline{P}(\varphi) \cdot \underline{Y}} \mathrm{~d} \Gamma$

In order to obtain a finite-dimension problem, this integral (22) can be discretized and one can consider the amplitude $w^{h}(\underline{X}, \underline{P}(\varphi))$ to be constant in each angular sector: $w^{h}\left(\underline{X}, \underline{P}\left(E_{P}\right)\right)$ (Fig. 4). The angular distributions of the plane waves for all points in the substructure are assumed to be well-described by this discontinuous angular distribution.

Once the discretization has been chosen for each plate, the VTCR leads to a system of linear equations in the complex domain:

$\mathbf{K}^{h} \underline{U}=\underline{F}^{h}$

where $\mathbf{K}^{h}=\mathbf{K}_{s}^{h}+\mathbf{Z}^{h}$ and $\underline{F}^{h}=\underline{L}_{D}^{h}$. $\mathbf{K}_{s}^{h}$ is the symmetric, positive definite damping matrix associated with $E_{D} ; \mathbf{Z}^{h}$ is the matrix associated with the bilinear form $\langle. .$.$\rangle defined such$ that $\mathbf{Z}^{h, T^{*}}=-\mathbf{Z}^{h} ; \underline{L}_{D}^{h}$ is the vector associated with the linear form $L_{D} ; \underline{U}$ is the vector corresponding to the unknown
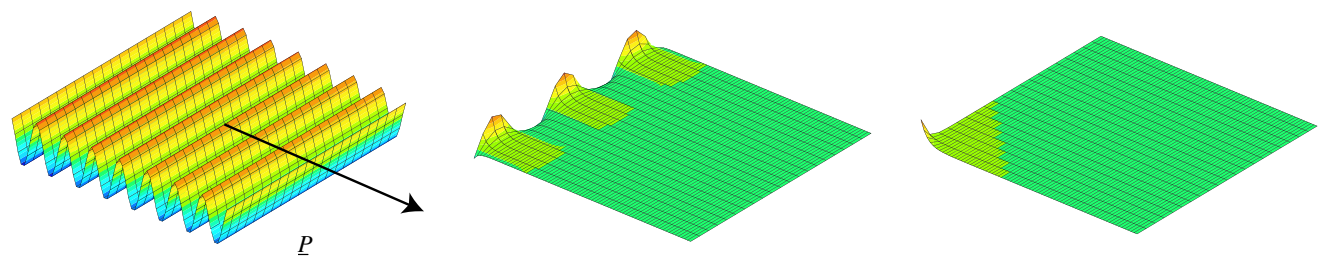

Fig. 3. Interior, edge and corner modes for a homogeneous plate. 

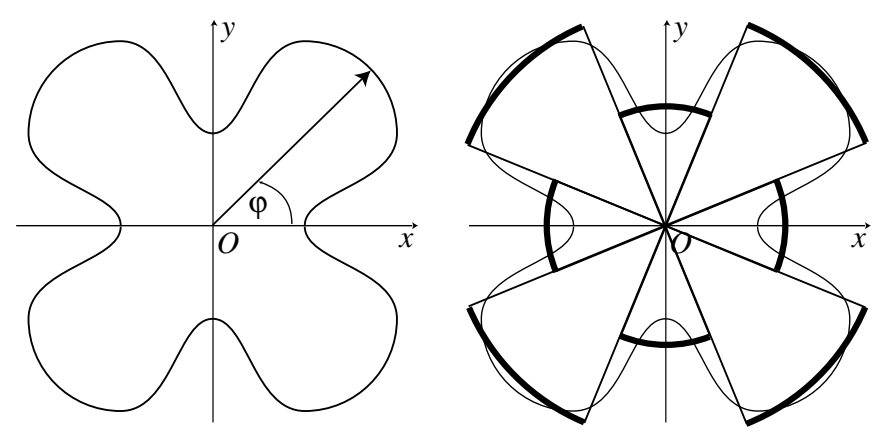

Fig. 4. The discretized amplitudes.

amplitudes associated with the complex polynomial $w^{h}\left(\underline{X}, E_{P}\right)$. As a consequence of the above properties, Eq. (23) has a unique solution. However, although the invertibility of matrix $\mathbf{K}$ (and, therefore, the uniqueness of the solution) has been proven, care is needed in its numerical calculation because this theory could lead to a poorly conditioned matrix $\mathbf{K}$ as in all Trefftz methods. In the VTCR, a dedicated numerical scheme, in which the fast variation terms are treated analytically $[24,25]$, is used to ensure sufficient accuracy.

\subsection{Effective quantities}

The spatial distribution of classical quantities such as the velocity $q(\underline{X})$ is meaningless from a mechanical point of view in the medium-frequency range because the response at a discrete location and at a discrete frequency is extremely sensitive to slight variations of input data. Thus, we retain only the effective quantities, which are spatially average quantities, defined on a domain greater than one wavelength. In the vicinity of a test point $X_{0}$, the associated effective quantities, which can represent the elastic energy, the kinetic energy or the dissipation work, are defined on a domain $S_{\underline{X_{0}}}$ by

$q_{\mathrm{eff}}\left(\underline{X_{0}}\right)=\frac{1}{S_{\underline{X_{0}}}} \int_{S_{\underline{X_{0}}}} q(\underline{X}) \mathrm{d} S$

These effective quantities are large-wavelength quantities and are much less sensitive.

\subsection{Effectiveness of the VTCR}

The capabilities of the method have been demonstrated on complex assemblies of plates [22-24]. Comparisons with industrial finite element codes showed that the VTCR is capable of predicting the effective quantities at a very low cost. The method was also extended to shells in [25] and heterogeneities were included in [26].

\subsection{The wide-frequency range analysis}

In our proposed approach to the transient dynamic response analysis, the Frequency Response Function (FRF) over a very wide-frequency range needs to be calcu- lated. Therefore, the ability to use the VTCR over a relatively wide-frequency range could be advantageous [20].

\subsubsection{Calculation of the VTCR in a frequency range}

Thus, the objective is to be able to calculate the solution in a frequency range $B$ with central frequency $\omega_{n}$ and bandwidth $\omega_{o}$ : Find $\underline{U}$ which verifies:

$$
\mathbf{K}^{h}(\omega) \underline{U}(\omega)=\underline{F}^{h}(\omega) \quad \forall \omega \in B=\left[\omega_{n}-\frac{\omega_{o}}{2} ; \omega_{n}+\frac{\omega_{o}}{2}\right]
$$

The idea is to introduce a two-scale approximation in terms of $\omega$. Any quantity $\alpha$, including the operator $\mathbf{K}^{h}(\omega)$ and the load $\underline{F}^{h}(\omega)$, can be written over $\omega \in B$ as

$\alpha(\omega)=\sum_{r=1}^{l} Q_{r}(\omega) A_{r}\left(\omega_{n}\right)$

where $A_{r}$ is assumed to be constant over $B . l$ is the angular sector and plays the same role as $h$-version of the finite element methods [24]. If necessary, one could build a better approximation for function $A_{r}$, e.g. a linear approximation. $Q_{r}$ is a rapidly varying function of $\omega$ equal to:

$Q_{r}(\omega)=\mathrm{e}^{2 \pi \mathrm{i} \omega q_{r}}$

where $q_{r}$ is one of the discrete values: $\{r \xi \mid r=-N,-N+$ $1, \ldots, 0,1,2, \ldots, N\}$.

In practice, $2 \omega_{c} N \xi$ is equal to the maximum number of space waves per substructure. $\xi$ or $N$ is a parameter which characterizes the quality of the two-scale approximation (26) $(N \sim 100-1000)$. Several techniques have been developed for determining an approximation of $\underline{U}$ in the form of (26) [27]. Here, we will introduce a new and quite efficient version [20].

Let us study the behavior of the exponential argument. This quantity can be expanded into a Taylor series up to order $\bar{k}$. The matrix $\mathbf{K}^{h}$ and load vector $\underline{F}^{h}$ can then be approximated as

$$
\begin{aligned}
\mathbf{K}^{h}(\omega) & =\sum_{k=0}^{\bar{k}} \mathbf{K}_{k}\left(\omega_{n}\right)\left(\omega-\omega_{n}\right)^{k} \\
\underline{F}^{h}(\omega) & =\sum_{k=0}^{\bar{k}} \underline{F}_{k}\left(\omega_{n}\right)\left(\omega-\omega_{n}\right)^{k}
\end{aligned}
$$

Let us also define the mean value over the frequency range $B$ :

$\langle\cdot\rangle=\frac{1}{\omega_{o}} \int_{B} \cdot \mathrm{d} \omega$

The matrix $\mathbf{K}^{h}$ and load vector $\underline{F}^{h}$ can be expressed as

$$
\begin{aligned}
& \mathbf{K}^{h}(\omega)=\left\langle\mathbf{K}^{h}\right\rangle+\Delta \mathbf{K}(\omega) \\
& \underline{F}^{h}(\omega)=\left\langle\underline{F}^{h}\right\rangle+\Delta \underline{F}(\omega)
\end{aligned}
$$

According to the Taylor expansion (27), one has

$$
\begin{aligned}
\Delta \mathbf{K}^{h}(\omega) & =\sum_{k=0}^{\bar{k}} \Delta \mathbf{K}_{k}\left(\omega_{n}\right)\left(\omega-\omega_{n}\right)^{k} \\
\Delta \underline{F}^{h}(\omega) & =\sum_{k=0}^{\bar{k}} \Delta \underline{F}_{k}\left(\omega_{n}\right)\left(\omega-\omega_{n}\right)^{k}
\end{aligned}
$$


$\underline{U}$ can also be defined using the same approach:

$\underline{U}^{h}(\omega)=\left\langle\underline{U}^{h}\right\rangle+\Delta \underline{U}(\omega)$

Over the frequency range $B$, Eqs. (25), (28) and (30) can be rewritten as

$\left[\left\langle\mathbf{K}^{h}\right\rangle+\Delta \mathbf{K}\right][\langle\underline{U}\rangle+\Delta \underline{U}]=\left\langle\underline{F}^{h}\right\rangle+\Delta \underline{F}$

Eq. (31) involves terms of very different magnitudes: some are large while others are small. Using the techniques of perturbation methods, different order terms can be identified and Eq. (31) can be rewritten for order 0 and order 1:

$\left.\left\langle\mathbf{K}^{h}\right\rangle \underline{U}\right\rangle=\left\langle\underline{F}^{h}\right\rangle \quad$ order 0

$\left\langle\mathbf{K}^{h}\right\rangle \underline{U}=-\Delta \mathbf{K}\langle\underline{U}\rangle+\Delta \underline{F} \quad$ order 1

with $\Delta \underline{U}$ equal to:

$\Delta \underline{U}=\sum_{k=0}^{\bar{k}} \Delta \underline{U}_{k}\left(\omega_{n}\right)\left(\omega-\omega_{n}\right)^{k}$

Finally, the displacement retained over the frequency range $B$ is

$\underline{U}=\lambda\langle\underline{U}\rangle+\mu \underline{\Delta}$

where $\lambda$ and $\mu$ minimize the error defined through the approximations in (32):

$E^{2}=\frac{1}{\omega_{o}} \int_{B}\left[\underline{F}^{h}-\mathbf{K}^{h} \underline{U}\right]^{\mathrm{T}}\left[\mathbf{K}_{s}^{h}\right]_{D}^{-1}\left[\underline{F}^{h}-\mathbf{K}^{h} \underline{U}\right] \mathrm{d} \omega$

$\left[\mathbf{K}_{s}^{h}\right]_{D}$ is the diagonal of the symmetric part of $\mathbf{K}^{h}$, which is a slowly varying function.

\section{Remarks}

- $\bar{k}$ corresponds to the degree of the Taylor expansion and is the only parameter that must be chosen to ensure good accuracy.

- The mean dissipated energy is

$$
e=\frac{\lambda^{2}}{2}\langle\underline{U}\rangle^{\mathrm{T}} \mathbf{K}_{s}^{h}\langle\underline{U}\rangle+\frac{\mu^{2}}{2 \omega_{o}} \int_{B} \Delta \underline{U}^{\mathrm{T}} \mathbf{K}_{s}^{h} \Delta \underline{U} \mathrm{~d} \omega
$$

- $\lambda, \mu$ can be $\omega$-functions.
This method over a frequency range was tested on beams and on assemblies of plates in [20]. For $\frac{\omega_{o}}{\omega_{n}} \approx 20 \%$, $\bar{k}=5$ seems to yield a very good approximation.

\section{Application on a complex structure}

\subsection{Reference problem}

The method is applied on an assembly of plates, the geometry of which is described in Fig. 5. This example corresponds to a typical stringer as can be found in a car chassis. The displacements of the structure are blocked on the right end and the structure is subjected to an impact load on one edge of the other end. The structure is damped with damping factor $\eta=0.02$.

The loading which characterizes the impact and its spectrum obtained through the Fourier transform are described in Fig. 6.

The first purpose of this example of a complex structure is to show the importance of the medium frequencies which, although the displacements are small, can play a significant role in the kinetic energy. It also aims at pointing out the efficiency of the approach.

\subsection{The method in the discrete frequency domain}

Let $T_{o}$ be the observation time also named extended period. It must be chosen large enough so that the response $f(t)$ fades out by the end of $T_{o}$ in order to avoid the overlapping in the time domain due to the use of the discrete Fourier transform and thus to obey causality. In practice $T_{o}$ is selected such that at the end of this period, the transient response is $\frac{1}{100}$ of the maximum value at the beginning. The input data as the loading are extended with zeros and the original problem defined on $[0, T]$ is now defined on $\left[0, T_{o}\right]$. Here the use of artificial damping in order to reduce $T_{o}$ is avoided because of the numerical oscillations that then appear in the response [28].

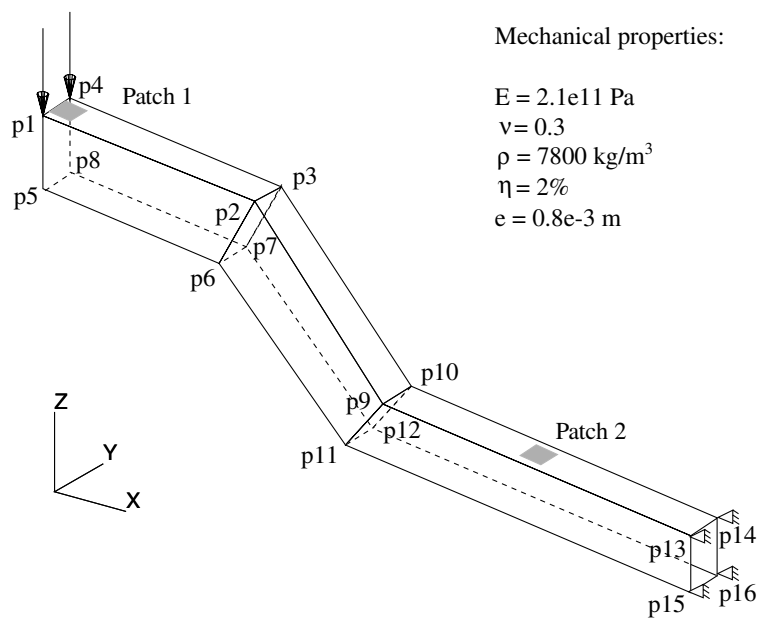

Points location (dimensions in meters):

Fig. 5. Assembly of 12 plates subjected to an impact loading. 

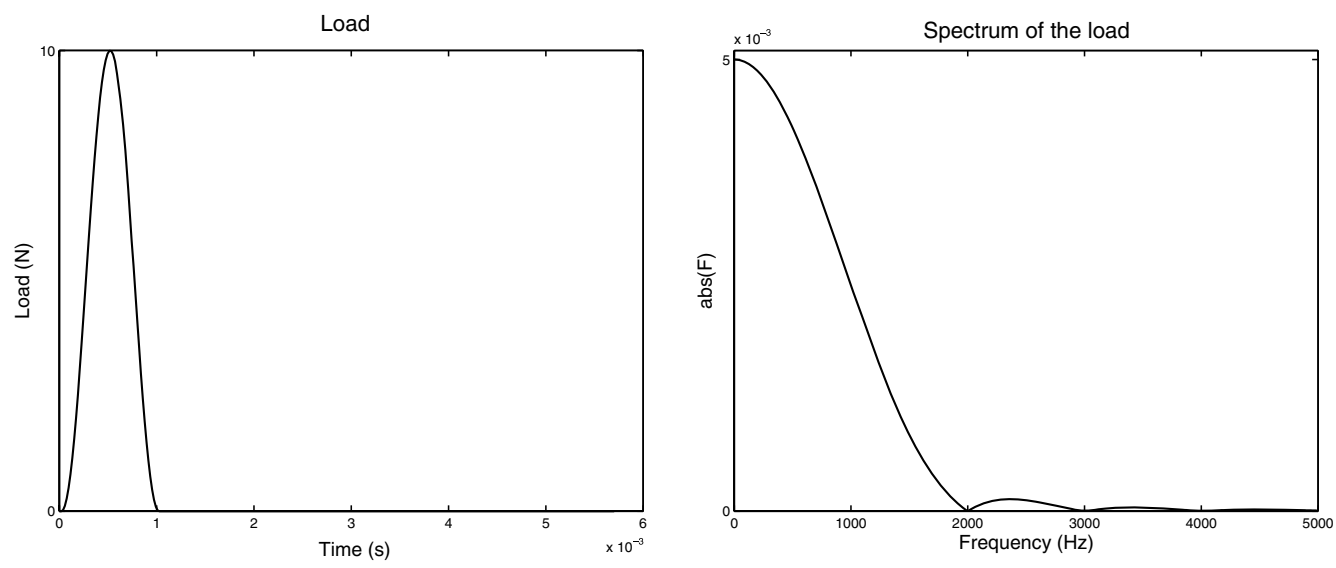

Fig. 6. The loading and its spectrum.

$N$ is the number of sampling points of the discrete Fourier transforms. $N$ must be a power of 2 in order to use the efficient fast Fourier transform (FFT) [29] and is chosen in respect with the law of at least seven time intervals $T_{e}=\frac{T_{o}}{N}$ to well describe the sine form of the impact load. $\omega_{n}=n \omega_{o}$ are the discrete frequencies where $\omega_{o}=\frac{2 \pi}{T_{o}}$.

Let $H\left(\omega_{n}\right)$ be the sampled frequency response function (FRF) over $\left[0, \omega_{c}^{\prime}\right]$ which is extended with zeros until the Nyquist frequency $\omega_{\text {Nyquist }}=\frac{N}{2} \omega_{o}$. Having calculated $H\left(\omega_{n}\right)$, we built the discrete Fourier transform $\hat{h}\left(\omega_{n}\right)$ of the unit response function $h(t)$ at the discrete frequency $\omega_{n}$ as in Eq. (37):

$\hat{h}\left(\omega_{n}\right)= \begin{cases}H\left(\omega_{n}\right) & \text { for } n \leqslant \frac{N}{2} \\ H^{*}\left(\omega_{N-n}\right) & \text { for } n>\frac{N}{2}\end{cases}$

The spectrum $\hat{f}\left(\omega_{n}\right)$ of the response $f(t)$ is determined by Eq. (38)

$\hat{f}\left(\omega_{n}\right)=\hat{h}\left(\omega_{n}\right) \hat{p}\left(\omega_{n}\right)$

where $\hat{p}\left(\omega_{n}\right)$ is the discrete Fourier transform of the impact load $p(t)$. Finally, we performed an inverse Fast Fourier Transform (iFFT) on $\hat{f}\left(\omega_{n}\right)$ in order to restore the timedependent response $f(t)$.

Before, carrying out the iFFT, $\hat{f}\left(\omega_{n}\right)$ was multiplied by a Hanning window in the frequency domain to avoid the Gibbs phenomenon once in the time domain.

\subsection{The frequency problem}

The frequency range $\left[0, \omega_{c}^{\prime}\right]$ being studied was divided into two parts: the low frequencies $\left[0, \omega_{c}\right]$ and the medium frequencies $\left[\omega_{c}, \omega_{c}^{\prime}\right]$.

\subsubsection{Choice of $\omega_{c}$}

As suggested in Section 3.2, a standard finite element technique was used to obtain the frequency response functions over the low-frequency range. $\omega_{c}$ was chosen such that the modes for this frequency and the higher frequen- cies become local modes, here $\omega_{c}=450 \mathrm{~Hz}$ and the corresponding solution of the forced vibration problem is shown in Fig. 7. This is also the frequency from which the modes are dense: in Fig. 8, the FRF of the effective speed over both patches of interest, of larger dimensions than the wavelengths of the medium frequencies, are plotted. It is therefore the frequency from which the accuracy of the computed FRF is highly mesh-dependent. In practice, $\omega_{c}$ is chosen by comparing the wavelength of the oscil-

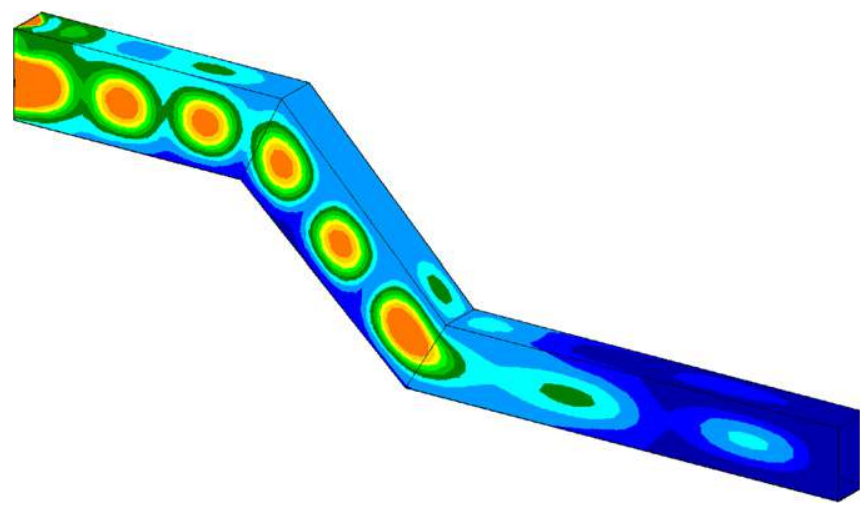

Fig. 7. Solution of the forced vibration problem at frequency $450 \mathrm{~Hz}$.

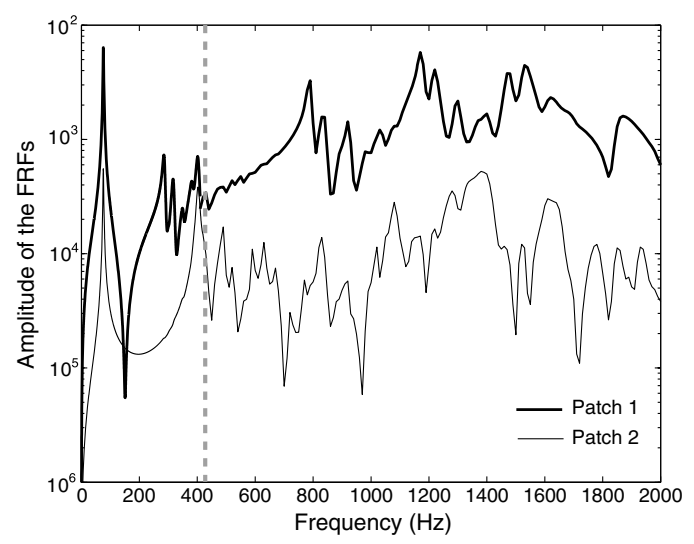

Fig. 8. FRF of the effective speed over the patches. 
lations at this frequency with the dimensions of the structure.

\subsubsection{Choice of $\omega_{c}^{\prime}$}

The frequency content of the response affects the velocity and, therefore, the kinetic energy to a great extent. Looking at Fig. 9 which shows the maximum of the kinetic energy in the two patches during the observation time as a function of the frequency range taken into account in the response, i.e. as a function of $\omega_{c}^{\prime}$, the frequency range necessary to derive the correct response was evaluated $\omega_{c}^{\prime}=\frac{2}{T}$, where $T$ is the characteristic time of the impact load. In our case $\omega_{c}^{\prime}=2000 \mathrm{~Hz}$. Looking at the spectrum of the impact load in Fig. 6, this criterion $\omega_{c}^{\prime}=\frac{2}{T}$ enables one to embrace most of the frequency content of the input signal.

In order to countenance this result, the velocities of two points, located on patch 1 and on patch 2, are plotted in Fig. 10. In these figures, the responses obtained with three different values of $\omega_{c}^{\prime}$ are compared.

As one can see, these graphics meet the results previously mentioned. Indeed the responses obtained with $\omega_{c}^{\prime}=2000 \mathrm{~Hz}$ and $\omega_{c}^{\prime}=3000 \mathrm{~Hz}$ are similar whereas the response with $\omega_{c}^{\prime}=1500 \mathrm{~Hz}$ varies from the other ones.

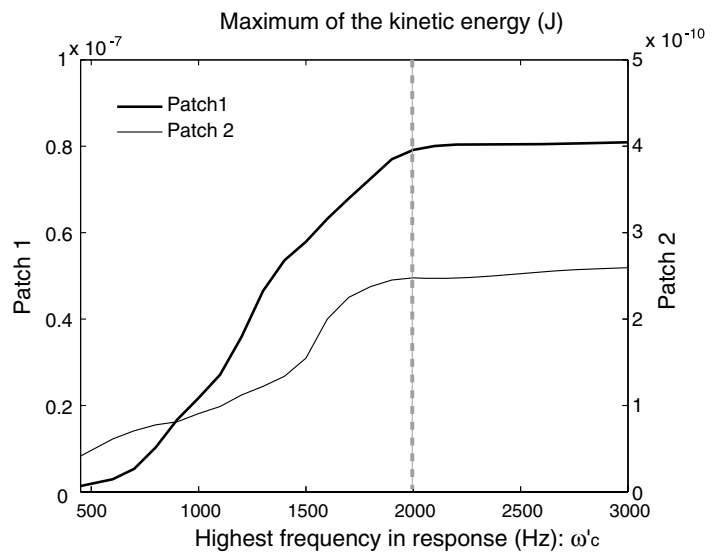

Fig. 9. Maximum of the kinetic energy in the patches during the observation time. $\omega_{c}^{\prime}=2000 \mathrm{~Hz}$ is actually a sufficient value of the upper bound of the medium-frequency (MF) range to derive the correct response.

The error done on the kinetic energy in the patches is less than $2 \%$ when taking into account the frequency range $[0-2000 \mathrm{~Hz}]$ in the transient response as can be seen in Fig. 11.

\subsubsection{The discrete frequency problem}

The problem to be solved was a discrete frequency problem since we used the iFFT. The frequency interval $\omega_{o}$ of the sampling was chosen such that $\omega_{o}=\frac{2 \pi}{T_{o}}$ where $T_{o}$ is the time by the end of which the waves fade out.

In order to reduce the number of frequency samples for the calculation of the frequency response function over the medium-frequency range, we took advantage of the fact that the studied structure is a damped scattering medium. Indeed the time taken by the waves to become negligible is much shorter for the medium frequencies than for the low frequencies because of the important dispersion of the medium-frequency bending modes.

Thus, in Eq. (39), the time response can be viewed as the superposition of two components, $f_{\text {low }}(t)$ and $f_{\text {mid }}(t)$,

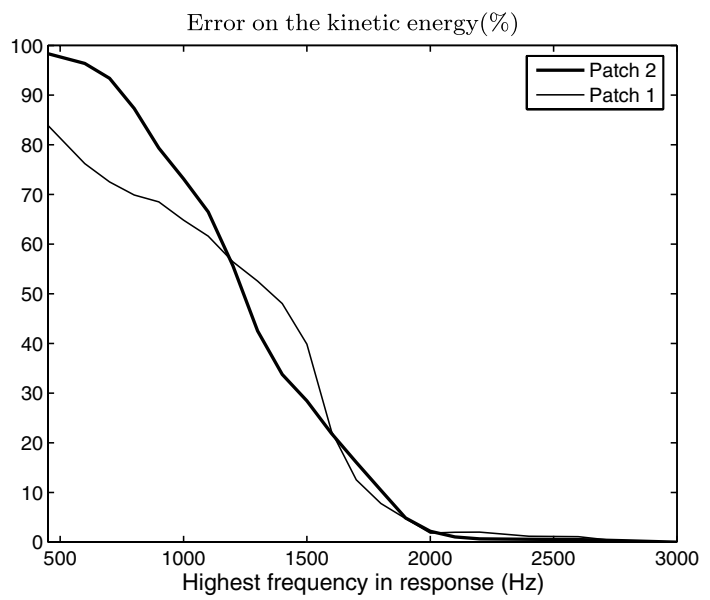

Fig. 11. Error on the kinetic energy in the patches as a function of $\omega_{c}^{\prime}$.

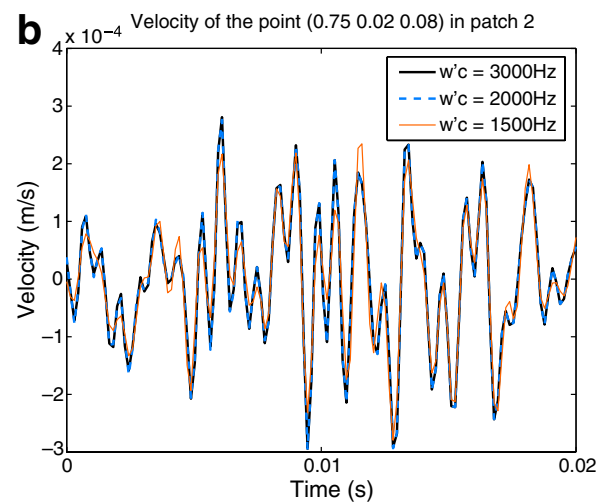

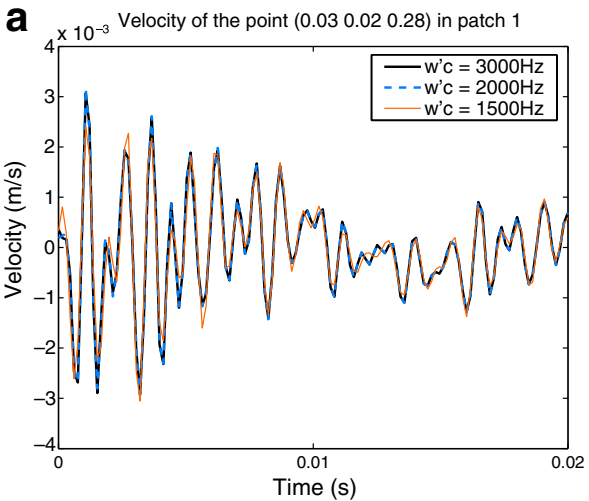

Fig. 10. Responses obtained with three different values of $\omega_{c}^{\prime}$ : (a) patch 1 and (b) patch 2. 
corresponding to the low-frequency contribution $\hat{f}_{\text {low }}(\omega)$ and the medium-frequency contribution $\hat{f}_{\text {mid }}(\omega)$ respectively

$$
\begin{aligned}
f(t)_{t \geqslant 0} & =\frac{1}{2 \pi} \int_{-\infty}^{+\infty} \hat{f}_{\text {low }}(\omega) \mathrm{e}^{\mathrm{i} \omega t} \mathrm{~d} \omega+\frac{1}{2 \pi} \int_{-\infty}^{+\infty} \hat{f}_{\text {mid }}(\omega) \mathrm{e}^{\mathrm{i} \omega t} \mathrm{~d} \omega \\
& =f_{\text {low }}(t)+f_{\text {mid }}(t)
\end{aligned}
$$

These two components were calculated separately with the frequency-domain approach presented in this paper and were added afterwards. Calculating the two components separately is convenient because in the calculation of $f_{\text {mid }}(t)$ the low frequencies are put aside and the medium frequencies alone are taken into account. Thus, one can take advantage of the rapid fading-out of these medium frequencies in the time response to shorten the observation time $T_{o}$ for the discrete Fourier transform to a considerable extent, which reduces the frequency sampling of the FRF $\hat{f}_{\text {mid }}$ over the medium-frequency range.

Since an inverse fast Fourier transform was performed, we used Hanning windows for both ranges to avoid the Gibbs phenomenon that would have occurred with a rectangle window. In order not to loose the amplitude of the

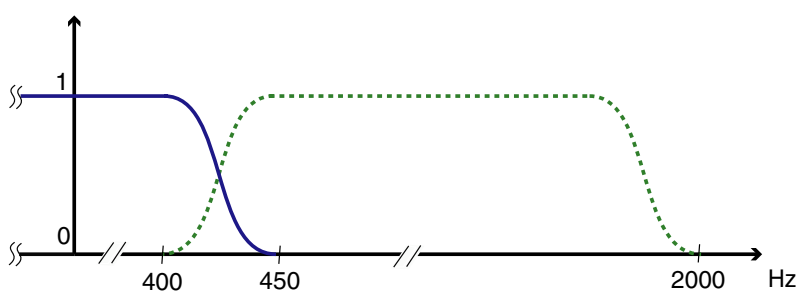

Fig. 12. Hanning windows of the two frequency ranges.

Table 1

Two frequency samplings for the MF range [400-2000 Hz]

\begin{tabular}{lll}
\hline & \multicolumn{2}{l}{ Discretization } \\
\cline { 2 - 3 } & 1 & 2 \\
\hline Observation time $T_{o}$ & $1 \mathrm{~s}$ & $0.1 \mathrm{~s}$ \\
$f_{o}=\frac{\omega_{o}}{2 \pi}$ & $1 \mathrm{~Hz}$ & $10 \mathrm{~Hz}$ \\
Number of calculations & 1601 & 161 \\
\hline
\end{tabular}

FRF around $\omega_{c}$, the two Hanning windows of the low and the medium-frequency ranges overlapped the common frequency range $[400-450 \mathrm{~Hz}]$ as illustrated in Fig. 12 .

The times needed for the waves to fade out in the studied structure were evaluated: $T_{o}=1 \mathrm{~s}$ for the low frequencies and $T_{o}=0.1 \mathrm{~s}$ for the medium frequencies. In order to verify that the time window $T_{o}=0.1 \mathrm{~s}$ is large enough to perform the iFFT on the medium-frequency contribution, two different time windows for these medium frequencies were used for comparison. On the one hand we took $T_{o}=0.1 \mathrm{~s}$ that enables one to take advantage of the rapid fading-out of the medium-frequency waves and thus to reduce the number of calculations, and on the other hand we took $T_{o}=1 \mathrm{~s}$ which is the time taken by the low-frequency waves to become negligible and consequently the default observation time chosen in our example when not taking advantage of the rapid fading-out of the medium frequencies. The choice of these two different time windows lead to the frequency samplings summarized in Table 1 and the corresponding responses are plotted in Fig. 13.

The responses are the same, we can therefore compute the two components separately and reduce the computational cost of the medium-frequency component. Finally, we retained for the two frequency ranges the frequency samplings proposed in Table 2.

Reducing the observation time, and thus the number of calculations, over the MF range is necessary especially if one were to extend the frequency-domain approach to nonlinear systems because studying such systems leads to the performance of many iFFT.

In the frequency-domain analysis procedure, the use of the discrete Fourier transform requires the FRF to be calculated for discrete frequencies $f_{n}=n f_{o}=n \frac{\omega_{o}}{2 \pi}$. But the solution at a discrete frequency being extremely sensitive to perturbations in the medium-frequency range, the frequency sampling can happen to be too coarse to well represent the peaks of the FRF.

In the aforementioned comparison, two different time windows, and consequently two different frequency samplings over the medium-frequency range were compared. In our example, the two different frequency discretizations
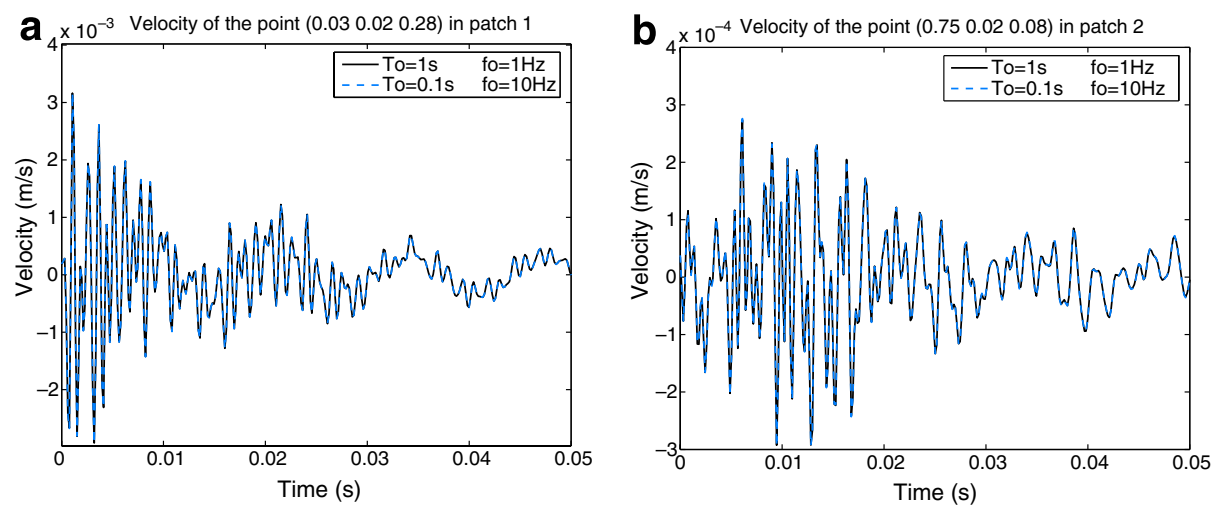

Fig. 13. Responses obtained with two different time windows for the MF range: (a) patch 1 and (b) patch 2. 
Table 2

Frequency sampling

\begin{tabular}{lll}
\hline & \multicolumn{2}{l}{ Frequency range } \\
\cline { 2 - 3 } & {$[0-450 \mathrm{~Hz}]$} & {$[400-2000 \mathrm{~Hz}]$} \\
\hline Time to fade out $T_{o}$ & $1 \mathrm{~s}$ & $0.1 \mathrm{~s}$ \\
$f_{o}=\frac{\omega_{o}}{2 \pi}$ & $1 \mathrm{~Hz}$ & $10 \mathrm{~Hz}$ \\
Number of calculations & 451 & 161 \\
\hline
\end{tabular}

lead to very similar results. Indeed, looking at the FRFs of the patches of interest of the studied structure, the minimum distance between two peaks is around $40 \mathrm{~Hz}$ in the medium-frequency range. Therefore, the coarser frequency sampling with $f_{o}=10 \mathrm{~Hz}$ appears to be sufficient to take the variations of the FRFs into account.

Anyway, to ensure that the variations of the FRF due to the high density of modes in the medium-frequency domain are taken into account, we can use the wide-frequency range analysis proposed in Section 4.6. This extent of the VTCR can be performed to enrich the frequency discretization with a low computational cost either by increasing the frequency sampling or by retaining, for each discrete frequency $\omega_{n}$ of the default frequency sampling, averaged quantities on a frequency range centered on $\omega_{n}$.

In the vicinity of a discrete frequency $\omega_{n}$, the associated averaged quantities are defined on a frequency range $B=\left[\omega_{n}-\frac{\omega_{o}}{2} ; \omega_{n}+\frac{\omega_{o}}{2}\right]$ by

$q_{\mathrm{eff}}\left(\omega_{n}\right)=\frac{1}{\omega_{o}} \int_{B} q(\underline{\omega}) \mathrm{d} \omega$

$q(\underline{\omega})$ over the frequency range $B$ is calculated thanks to the large frequency range analysis of the VTCR and enables to account for the content and the variations of the FRF.

In the present example, all the results were obtained without using this large frequency range analysis of the VTCR since it is not yet implemented in the dedicated software CORAY MF.

\subsection{The spatial discretized problem of the frequency-domain approach}

\subsubsection{Space discretization of the low-frequency problem}

A standard finite element technique, performed with MSC.Nastran, was used to obtain the frequency response functions over the low-frequency range. $\omega_{c}$ was chosen $\omega_{c}=450 \mathrm{~Hz}$. In order to derive a proper FRF, the mesh was constructed with seven linear elements per wavelength of the upper bound of the low-frequency range. The reduced basis was constructed from the first 50 modes to arrive at a highest eigenfrequency $\omega_{50}=2 \omega_{c}$, and was completed with the static bending mode.

If one were to calculate the FRF over $\left[0, \omega_{c}^{\prime}\right]$, it would have required 336,000 degrees of freedom (DOFs) in respect with the law of seven elements per wavelength and 500 modes in the reduced basis would have been necessary to derive the correct FRF over [0-2000 Hz].
Table 3

Characteristics of the mesh in MSC. Nastran for the computation of the FRF

\begin{tabular}{lll}
\hline & \multicolumn{2}{l}{ Frequency range } \\
\cline { 2 - 3 } & {$[0-450 \mathrm{~Hz}]$} & {$[0-2000 \mathrm{~Hz}]$} \\
\hline Number of DOFs & 19,700 & 336,000 \\
Reduced basis & 50 modes & 500 modes \\
\hline
\end{tabular}

Carrying out such calculation is prohibitive and can also lead to numerical difficulties.

Table 3 summarizes the space discretized problems in MSC.Nastran for the calculation of the FRF over [0$450 \mathrm{~Hz}]$ on the one hand and over $[0-2000 \mathrm{~Hz}]$ on the other hand.

\subsubsection{Space discretization of the medium-frequency problem}

For complex structures, the VTCR turned out to be a suitable computational method for the medium-frequency range: it is very efficient because its multiscale point of view leads to use a very low number of DOFs even at high frequencies. Ref. [24] shows that when the frequency increases, the use of a classical finite element approach leads to huge numbers of DOFs whereas the VTCR enables one to use a constant mesh size maintaining very good accuracy while decreasing the CPU time.

For the VTCR, the number of substructures and of rays needed per substructures is selected as advised in [24]. Here, the structure was divided into 12 substructures which correspond to the 12 plates of the assembly. Thirty-two interior modes and 5 edge modes per edge were used per substructure. The computation of the medium-frequency range was performed with the dedicated software CORAY MF. Table 4 summarizes the spatial discretization of the medium-frequency range.

\subsection{Contribution of the medium frequencies}

The feasibility of the method and the importance of the medium frequencies in transient dynamics have been pointed out on academic examples made of assemblies of beams in [3]. In the absence of the medium frequencies, the small oscillations due to these frequencies, the kinetic energy of which is very significant, are killed.

Same conclusions can be made for more complex structures.

Indeed, looking at the FRFs of the two patches plotted in Fig. 8, we can notice that, as expected, the first peak of the FRFs, plotted in Fig. 8, at $\omega=75.6 \mathrm{~Hz}$, is the global bending mode (see Fig. 14) which is highly excited by the chosen impact load. But we can also notice that many

Table 4

Characteristics of the space discretization with the VTCR

\begin{tabular}{ll}
\hline Frequency range & {$[450-2000 \mathrm{~Hz}]$} \\
Number of substructures & 12 \\
Number of rays (DOFs) & 624 \\
\hline
\end{tabular}

Number of rays (DOFs) 624 


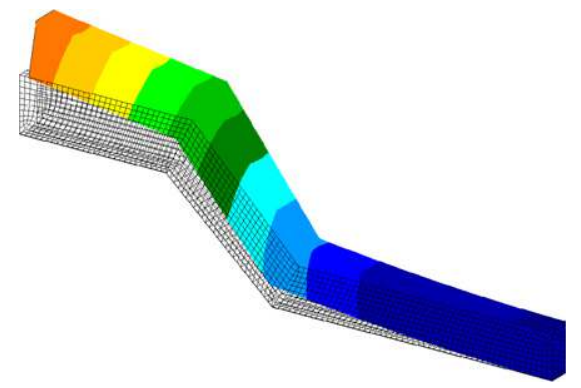

Fig. 14. Mode at frequency $75.6 \mathrm{~Hz}$.

higher frequencies contribute to a great extent in the content of the FRF. Indeed, looking at the local aspect of the impact load, medium-frequency modes which are local modes are excited. The most important excited modes are illustrated Fig. 15.

In this example, almost all of the kinetic energy induced in patch 1 , situated near the impact load, is due to the medium frequencies as can be noticed in Fig. 9. As an example, the velocity of a point located in this patch is plotted in Fig. 16. The low-frequency part of the velocity is plotted with a light grey color in order to highlight the importance of the medium-frequency content.

These medium frequencies damp more rapidly than the low-frequency waves: indeed the relative proportion between the medium-frequency content and the low-frequency content is lower in patch 2 , situated further from the impact load, than in patch 1 as can be noticed in Figs. 9 and 17.

\subsection{Advantage of the frequency-domain method}

The advantage of the frequency-domain method is that the solution is explicitly known over the whole time. Indeed, the discretized problem described in Table 2

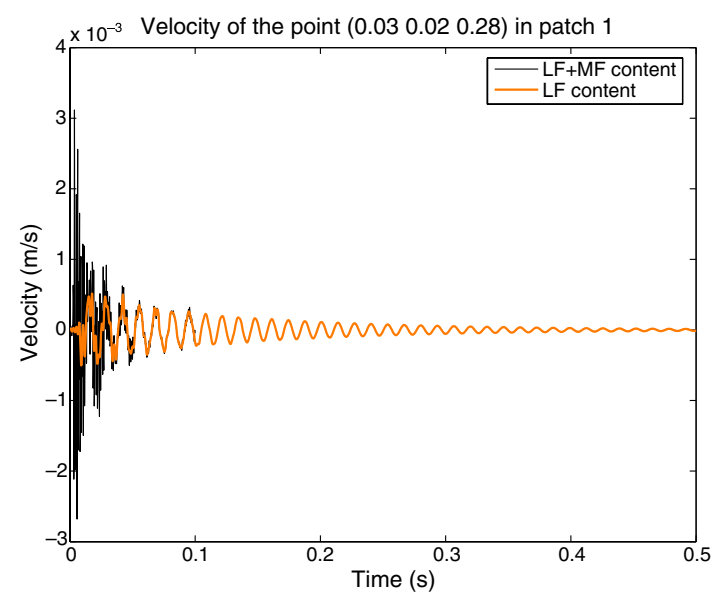

Fig. 16. Velocity of a point located in the patch 1.

enables one to compute the response of the structure for a large time interval until the transient response dies out, here $T_{o}=1 \mathrm{~s}$.

As for time domain methods that use numerical schemes, the transient response is known step by step and they require at least 7000 time steps to compute the response of the structure studied here: the number of time steps is evaluated according to the law of at least seven time steps to well describe the sine form of the impact load and 7000 time steps unable to compute the response over the time period necessary for the velocity to fade out. Furthermore the required mesh would have been much more refined in each direction which would have lead to 336,000 degrees of freedom and consequently to numerical difficulties [5].

For such complex structures, the construction of a reduced basis sufficiently rich in modes to deal with fast dynamics, such as shocks, is often unfeasible; therefore,
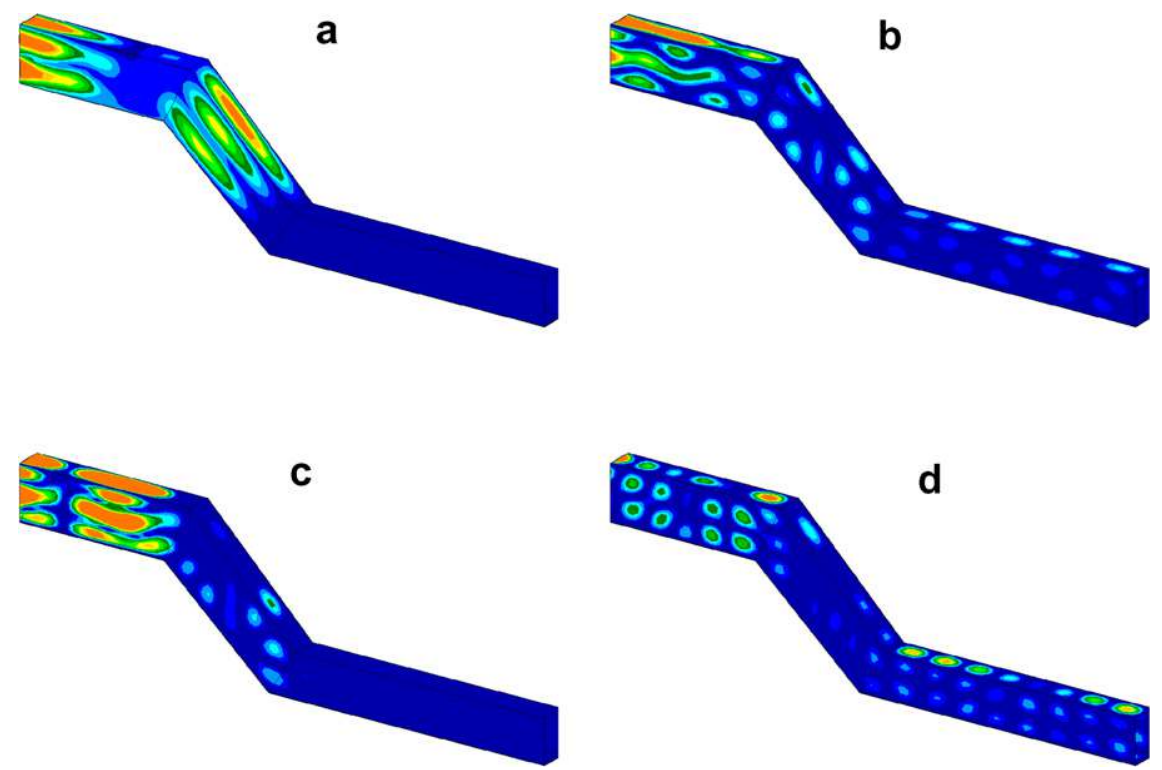

Fig. 15. Higher local modes that contribute in the response: (a) $790 \mathrm{~Hz}$, (b) $1170 \mathrm{~Hz}$, (c) $1220 \mathrm{~Hz}$ and (d) $1470 \mathrm{~Hz}$. 


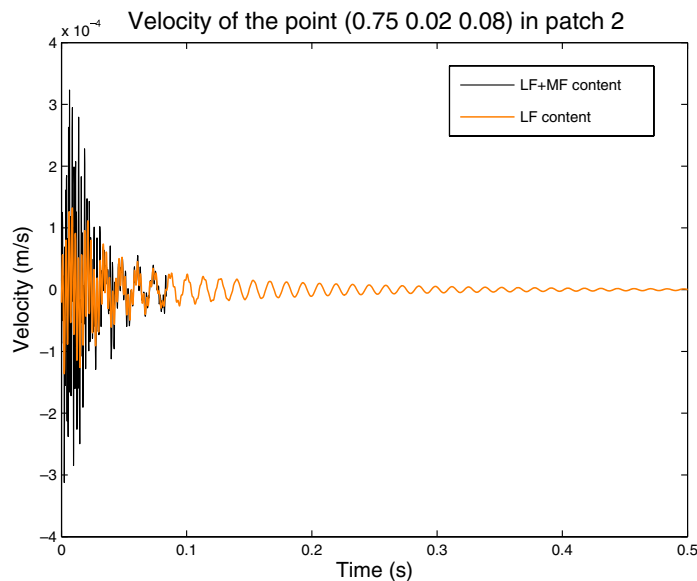

Fig. 17. Velocity of a point located in the patch 2.

Table 5

Sizes of the computational problems for the time-domain (TD) and the frequency-domain (FD) approaches over $T_{o}=1 \mathrm{~s}$

\begin{tabular}{|c|c|c|c|}
\hline \multirow[t]{2}{*}{ Approach } & \multirow{2}{*}{$\begin{array}{l}\text { TD } \\
\text { Reduced } \\
\text { basis }\end{array}$} & \multicolumn{2}{|l|}{ FD } \\
\hline & & $\begin{array}{l}\text { Reduced } \\
\text { basis }\end{array}$ & VTCR \\
\hline DOFs & 336,000 & 19,700 & \\
\hline Eigenmodes & 500 & 50 & \\
\hline Complex rays & & & 624 \\
\hline Number of calculations & 7000 & 451 & 161 \\
\hline $\begin{array}{l}\text { Size of the computational } \\
\text { problem }\end{array}$ & $7000 \times 500$ & \multicolumn{2}{|c|}{$451 \times 50+161 \times 624$} \\
\hline CPU-time & $43 \mathrm{~h}$ & \multicolumn{2}{|l|}{$1 \mathrm{~h}$} \\
\hline
\end{tabular}

in these cases, the time integration is carried out on the finite element matrices directly: this leads to even greater computation times.

In our method, the frequency content is extended using only a few VTCR calculations. The frequency-domain analysis procedure also requires discrete Fourier transforms, which are performed using the iFFT algorithms. The use of the iFFT makes the frequency approaches efficient for the analysis of the dynamic response of linear systems [30].

In the case of nonlinear systems, many iFFT need to be performed. Having reduced the amount of VTCR calculations as explained in Section 5.3.3 is then critical.

Finally, the costs of a classical time-domain approach, using a finite element mesh and an explicit numerical scheme, and the proposed frequency-domain approach for the studied linear example are compared in Table 5:

- the time-domain approach requires at least 7000 time steps and a refined finite element mesh of $336 \times 10^{3}$ DOFs. In order to take the frequencies up to $2000 \mathrm{~Hz}$ into account, the 500 first eigenmodes need to be computed for the use of a reduced basis;

- in the frequency-domain approach, the FRF is predicted by solving 451 forced vibration problems with a small reduced basis constructed from the 50 first eigenmodes for the low-frequency range [0-450 Hz], and by solving only 161 forced vibration problems for the medium frequencies [450-2000 Hz] thanks to the VTCR using 624 rayons complexes.

\section{Conclusion}

We have developed a new robust method for the calculation of transient dynamic responses with a high frequency content for a rather complex structure made of plates that tend towards engineering structures. The method is now to be applied on assemblies of shells, plates and beams with heterogeneities. The point of this technique is to restitute both the low and especially the medium frequencies that easily occur in complex structures while decreasing the CPU-time. Moreover with this approach, the transient response is explicitly known on the whole time observation. The extension to nonlinearities expressed in the time domain and to 3D-problems is the subject of further developments.

\section{References}

[1] Bathe KJ. Finite element procedures. 2nd ed.: Prentice-Hall; 1995.

[2] Belytschko T, Liu W, Moran B. Nonlinear finite elements for continua and structures. John Wiley \& Sons; 2000.

[3] Ladevèze P. A new computational method for medium-frequency vibrations and its extension to transient dynamics. In: Owen DRJ, Oñate E, Suárez B, editors. Proceedings of the seventh international conference on computational plasticity - Complas 2003, CIMNE, Barcelona, Spain, on CD-ROM, 7-10 April 2003.

[4] Lyon R, Maidanik G. Power flow between linearly coupled oscillators. JASA 1962;35(5):623-39.

[5] Ilhenburg F, Babuška I. Dispersion analysis and error estimation of Galerkin finite element methods for Helmholtz equation. Int J Numer Methods Engrg 1995;38:3745-74.

[6] Deraemaeker A, Babuška I, Bouillard P. Dispersion and pollution of the FEM solution for the Helmholtz equation in one, two and three dimensions. Int J Numer Methods Engrg 1999;46:471-99.

[7] Farhat C, Harari I, Hetmaniuk U. A discontinuous Galerkin method with Lagrange multipliers for the solution of Helmholtz problems in the mid-frequency regime. Comput Methods Appl Mech Engrg 2003;192:1389-419.

[8] Harari I, Haham S. Improved finite element method for elastic waves. Comput Methods Appl Mech Engrg 1998;166:143-64.

[9] Liu WK, Zhang Y, Ramirez MR. Multiple scale finite element methods. Int J Numer Methods Engrg 1991;32:969-90.

[10] Morand JPH. A modal hybridization method for the reduction of dynamic models. In: Ladevèze $\mathrm{P}$, Zienkiewicz $\mathrm{O}$, editors. New advances in computational structural mechanics. Elsevier; 1992. p. $347-65$.

[11] Soize C. Reduced models in the medium frequency range for general dissipative structural-dynamics systems. Eur J Mech A/Solids 1998;17(4):657-85.

[12] Sarka A, Ghanem R. Mid-frequency structural dynamics with parameter uncertainty. Comput Methods Appl Mech Engrg 2002;191:5499-513.

[13] Desmet W, Van Hal B, Sas P, Vandepitte D. A computationally efficient prediction technique for the steady-state dynamic analysis of coupled vibro-acoustic systems. Adv Engrg Softw 2002;33:527-40.

[14] Belov VD, Ryback SA. Applicability of the transport equation in the one-dimensional wave propagation problem. Akust Zh Sov Phys Acoust 1975;21/2:173-80. 
[15] Ichchou MN, Le Bot A, Jézéquel L. Energy model of onedimensional, multipropagative systems. J Sound Vib 1995;201(5): $535-554$.

[16] Langley RS. On the vibrational conductivity approach to high frequency dynamics for two-dimensional structural components. J Sound Vib 1995;182 (4):637-57.

[17] Lase Y, Ichchou MN, Jézéquel L. Energy flow analysis of bars and beams: theoretical formulation. J Sound Vib 1994;192(1):2981-3005.

[18] Ladevèze P. A new computational approach for structure vibrations in the medium frequency range. CR Acad Sci Paris Sér II 1996; 322(12):849-56.

[19] Ladevèze $\mathrm{P}$, Chevreuil $\mathrm{M}$. A new computational method for transient dynamics including the low- and the medium-frequency ranges. Int $\mathbf{J}$ Numer Methods Engrg 2005;64:503-27.

[20] Ladevèze P, Riou H. Calculation of medium-frequency vibrations over a wide frequency range. Comput Methods Appl Mech Engrg 2005;194(27-29):3167-91.

[21] Zienkiewicz O, Taylor R. The finite element method. 4th ed. London: McGraw-Hill; 1984.

[22] Ladevèze P, Arnaud L. A new computational method for structural vibrations in the medium frequency range. Comput Ass Mech Engrg Sci 2000;7:219-26.
[23] Ladevèze P, Arnaud L, Rouch P, Blanzé C. The variational theory of complex rays for the calculation of medium-frequency vibrations. Engrg Comput 2001;18:193-214.

[24] Rouch P, Ladevèze P. The variational theory of complex rays: a predictive tool for medium-frequency vibrations. Comput Methods Appl Mech Engrg 2003;192:3301-15.

[25] Riou H, Ladevèze $P$, Rouch $P$. Extension of the variational theory of complex rays to shells for medium-frequency vibrations. J Sound Vib 2004;272:341-60.

[26] Ladevèze P, Blanc L, Rouch P, Blanzé C. A multiscale computational method for medium-frequency vibrations of assemblies of heterogeneous plates. Comput Struct 2003;81(12):1267-76.

[27] Ladevèze P, Rouch P, Riou H, Bohineust X. Analysis of mediumfrequency vibrations in a frequency range. J Comput Acoust 2003; 11(2):255-83.

[28] Humar JL, Xia H. Dynamic response analysis in the frequency domain. Earthquake Engrg Struct Dynam 1993;22:1-12.

[29] Brigham EO. The fast Fourier transform and its applications. Englewood Cliffs, NJ: Prentice-Hall, Inc.; 1988.

[30] Clough RW, Penzien J. Dynamics of structures. 2nd ed. Education: McGraw-Hill; 1993. 